\title{
BMJ Open Exploring goal planning in mental health service delivery: a systematic review protocol
}

\author{
Victoria Stewart (D) , , Sara Sinclair McMillan (1) , ${ }^{1}$ Helena Roennfeldt (D) ,2,4 \\ Sarira El-Den (1) , ${ }^{5}$ Ricki Ng (D) , ${ }^{5}$ Amanda J Wheeler (1) ${ }^{2,6}$
}

To cite: Stewart V, McMillan SS, Roennfeldt $\mathrm{H}$, et al. Exploring goal planning in mental health service delivery: a systematic review protocol. BMJ Open 2021;11:e047240. doi:10.1136/ bmjopen-2020-047240

- Prepublication history for this paper is available online. To view these files, please visit the journal online (http://dx.doi org/10.1136/bmjopen-2020047240).

Received 22 November 2020 Accepted 09 March 2021

\section{Check for updates}

(c) Author(s) (or their employer(s)) 2021. Re-use permitted under CC BY-NC. No commercial re-use. See rights and permissions. Published by BMJ.

${ }^{1}$ School of Human Services and Social Work, Griffith University, Meadowbrook, Queensland,

Australia

${ }^{2}$ Menzies Health Institute, Griffith University, Nathan, Queensland, Australia

${ }^{3}$ School of Pharmacy and Pharmacology, Griffith University Faculty of Health, Gold Coast, Queensland, Australia

${ }^{4}$ Centre for Psychiatric Nursing, The University of Melbourne, Carlton, Victoria, Australia

${ }^{5}$ Pharmacy, The University of Sydney, Sydney, New South

Wales, Australia

${ }^{6}$ Faculty of Health and Medical

Sciences, The University of Auckland, Auckland, New Zealand

Correspondence to Ms Victoria Stewart; v.stewart@griffith.edu.au

\section{ABSTRACT}

Introduction Goal planning is widely recognised as an integral part of mental health service delivery and an important element in supporting recovery. Goal planning identifies priorities for treatment through discussion and negotiation between service users and health practitioners. Goal planning enhances motivation, directs effort, and focuses the development of strategies and treatment options to improve recovery outcomes and promote service users' ownership of the recovery process. While goal planning is a common practice in mental health settings, evidence regarding its impact on treatment outcomes is lacking. This paper outlines a protocol for a systematic review that aims to explore the types of goals planned, experiences of service users and practitioners, and the effectiveness of goal planning as a mental health intervention.

Methods and analysis A systematic search will be conducted during March 2021 by searching Medline, CINAHL, Embase, Scopus and PsycINFO electronic databases to answer the following questions: (1) What types of goals are being developed within mental healthcare?; (2) What is the evidence for the effectiveness of goal planning on health and well-being for mental health service users?; (3) What are the experiences of mental health service users and their treating healthcare practitioners in relation to goal planning?; and (4) What are the barriers and facilitators to effective goal planning in mental health settings? Two independent researchers will screen the articles, selecting literature that meets criteria. All literature, regardless of study design that involves adult participants, with a mental illness and reporting on goal planning will be considered for inclusion. Data will be extracted from all eligible articles regardless of study design and summarised in a table. Appropriate quality assessment and data synthesis methods will be determined based on included study designs.

Ethics and dissemination No ethics approval is required. The results will be disseminated through peer-reviewed publications and conference presentations. PROSPERO registration number CRD42020220595.

\section{INTRODUCTION}

Identifying and writing a goal is a familiar process to most people, improving the likelihood of achieving a desired outcome, and assisting us to accomplish and achieve

\section{Strengths and limitations of this study}

- This is the first systematic review focusing on the types of goals being set, experiences of service users and practitioners, and the effectiveness of goal planning as a mental health intervention.

- Study selection will be undertaken by two researchers to ensure all relevant studies are included without the risk of personal biases.

- All relevant studies will be included in the review, regardless of study design, to provide a deeper understanding of the impact of contextual factors and to map diverse measurements of effect.

- Appropriate synthesis of the data may be challenging given the diversity in study designs but will allow for a holistic understanding of the topic through the integration of findings from multiple sources of data.

- This review will only include studies published in English which may result in cultural and language bias.

throughout our lives. ${ }^{12}$ Goal planning has an important role in the rehabilitation of adults with a disability (eg, following brain injury, mental illness or muscular injuries). ${ }^{3}$ Goal planning within healthcare and rehabilitation settings has been found to have a positive effect on health-related quality of life, selfreported emotional status and service user self-efficacy. ${ }^{3}$ A number of psychological theories underpin the process of goal planning and describe how setting goals allows people to monitor, alter or adapt their behaviour. ${ }^{4}$ Social cognitive theory ${ }^{5}$ emphasises the influence of self-efficacy on an individual's motivation to set and achieve goals, while health action theory ${ }^{6}$ highlights the phases involved in goal planning and achievement. Goal setting theory ${ }^{7}$ identifies the factors which impact on the effectiveness of goal planning including the specificity and difficulty of goals set and the influence of goal commitment, self-efficacy, task complexity and performance feedback on goal effects. 
Goal planning is generally understood as a process of discussion and negotiation through which service users and health practitioners identify priorities for treatment to achieve a desired future state. ${ }^{8}$ How goals are used in healthcare and rehabilitation settings is not always clearly defined and a range of terminology and approaches to goal planning have been described within the literature. ${ }^{3}$ For the purpose of this review, the term goal planning will be used as it is commonly used in mental health recovery literature, encompassing other terms including goals, goal setting and goal attainment. To support positive outcomes, it is generally agreed that goals should be collaboratively developed, relevant to the person concerned, challenging but realistic and achievable, and include a component of measurability. ${ }^{9}{ }^{10}$ Goal planning is used extensively within many healthcare settings and the use of goals is central in mental health service delivery and is a well-regarded component of psychiatric rehabilitation. ${ }^{11}$

Recovery is now an established concept that underpins international mental health policy and service provision and was developed by service users to counter the dominant biomedical discourse of chronic, enduring mental illness. ${ }^{12}{ }^{13}$ While there is no universally accepted definition of recovery, it has been described as 'a deeply personal, unique process of changing one's attitudes, values, feelings, goals, skills, and/or roles ... a way of living a satisfying, hopeful, and contributing life even with limitations caused by illness. ${ }^{14}$ This definition of recovery contrasts strongly with more traditional medical models of recovery that focus on outcomes such as decreased symptomatology, hospitalisations and medications. ${ }^{15}$ Due to the influence of recovery, goal planning for people experiencing mental illness is likely to be different from other healthcare settings. Recovery-oriented practitioners aim to support a person to achieve their personal aims, desires, hopes and dreams in life, assisting them to identify goals or outcomes and preferred interventions to achieve these. ${ }^{16}$ The pursuit of personal goals is an important recovery strategy and it is therefore essential that goal planning be considered in relation to psychiatric rehabilitation. ${ }^{17}$

While considerable attention has been given to researching the process of goal planning in rehabilitation generally, ${ }^{3} 1018$ there has been limited review of the literature regarding the use of goal planning in mental health service delivery. Jørgensen and Rendtorff ${ }^{19}$ identified shared decision-making and equal collaboration between mental health practitioners and service users as important in the setting of recovery-oriented goals. In contrast to traditional medical or authoritative models of healthcare, shared decision-making is a collaborative, dynamic and interactive process between equally involved parties, exchanging information to agree on treatment priorities and processes. ${ }^{20}$ Service users who experience goal planning that is a mutual or shared process perceive their goals to be more relevant, have a better understanding of, and engagement in the rehabilitation process. ${ }^{21} 22$ Shared decision-making processes have been found to result in higher self-efficacy in mental health service users and a more positive attitude towards medication. ${ }^{20}$

While policies increasingly support the inclusion of service users in goal planning, implementation lags behind and concerns regarding how to set goals that service users find personally meaningful have been noted. ${ }^{23}{ }^{24}$ Research suggests that although health practitioners generally support the principles of shared decision-making, in practice they tend to focus on the reduction of symptoms, providing expert advice regarding diagnosis, course of illness and treatment options. ${ }^{25}{ }^{26}$ In contrast, service users emphasise broad, long-term goals that express their hopes and aspirations, prioritising goals related to non-medical concerns such as managing relationships, emotions and practical challenges such as housing and employment. ${ }^{2326}$ In addition, if the service user is assessed as experiencing severe mental health symptoms, they may be seen as lacking capacity, with practitioners less likely to involve them in shared decision-making processes. ${ }^{19} 28$

While goal planning is increasingly being used within mental health service delivery, there is no clear understanding of the types of goals being set, experiences of service users and practitioners, or the effectiveness of goal planning as a mental health intervention. A systematic review can facilitate the identification of literature exploring the effect of goal planning in mental health service delivery, and the experiences of key stakeholders, thereby providing guidance on how goal planning should be used in mental health service delivery, identifying knowledge gaps and providing recommendations for future research.

\section{Research questions}

The aim of this systematic review is to explore the literature pertaining to goal planning within mental health service delivery from the perspective of health practitioners and service users, specifically:

- What types of goals are being developed within mental health service delivery?

- Is goal planning effective in improving the health and well-being of mental health service users?

- What are the experiences of mental health service users and their treating healthcare practitioners in relation to goal planning?

-What are the barriers and facilitators to effective goal planning in mental health service delivery from the perspectives of health practitioners and service users?

For the purpose of the review, the definition of a rehabilitation goal by Siegert and Levack will be applied, that is, 'Rehabilitation goals are actively selected, intentionally created, have purpose and are shared (where possible) by the people participating in the activities and interventions designed to address the consequence of acquired disability' with goal planning defined as 'the establishment or negotiation of rehabilitation goals' $(\mathrm{p} 11){ }^{8}$ 


\begin{tabular}{|c|c|}
\hline PICO & Search terms \\
\hline $\mathbf{P}$ & $\begin{array}{l}\text { "mental disorder"[MeSH] OR "mental health"[MeSH] OR "mental illness"” OR "mental health" OR "mental disorder" } \\
\text { OR "mental health condition*" OR "mental health diagnosis" OR "psychiatric illness"” OR "psychiatric disorder" OR } \\
\text { "psychiatric condition*" OR "psychiatric diagnosis" OR "mental health consumer" OR "mental health service user"” } \\
\text { NOT child* OR youth* OR adolescent" }\end{array}$ \\
\hline I & $\begin{array}{l}\text { AND } \\
\text { (“Goals" [MeSH] OR Goal*) OR (“Patient Care Planning”[MeSH] OR (Care AND plan*)) OR (“Decision making, } \\
\text { shared"[MeSH] OR "shared decision mak*”) } \\
\text { AND } \\
\text { (set* OR plan* OR attain* OR achieve* OR assess* OR orient* OR commit* OR "action plan" OR "recovery plan") } \\
\text { Note: search terms will be modified to suit individual databases for example, Emtree or Subject Headings }\end{array}$ \\
\hline 0 & No search limitations imposed. \\
\hline
\end{tabular}

MeSH, Medical Subject Headings; PICO, population, intervention, comparison group, outcome.

\section{METHODS AND ANALYSIS}

The systematic review process has been developed in accordance with recommendations from the Preferred Reporting Items for Systematic Review and Meta-Analysis Protocols, ${ }^{29}$ presented in the online supplemental file. The protocol has been registered with the International Prospective Register of Systematic Reviews.

\section{Search strategy}

Medline (Ebsco), CINAHL, Embase (Embase.com), Scopus and PsycINFO (Ovid) electronic databases will be searched in March 2021. Once eligible documents identified, Google Scholar will be used for forward/backward citation checking. An academic librarian will be consulted for the validation of the databases and the final search terms. Terms will be searched as free-text terms or as index terms (eg, Medical Subject Headings) where appropriate.

The search will target studies reporting on the use of goal planning with adult mental health service users using keywords and subject headings. Search terms are outlined below using the PICO framework (table 1). The PICO framework is commonly used in evidencebased healthcare and was adapted and used to structure the search strategy. ${ }^{30}$ ' $\mathrm{P}$ ' refers to the population (adults experiencing mental illness); 'I' refers to the intervention (goal planning); 'C' refers to comparison group (which is not relevant for this study) and ' $\mathrm{O}$ ' refers to outcome. As this study is interested in understanding a range of outcomes, no search term limitations will be used.

There will be no date restriction set for study/literature publication or study time frame, and all mental health settings (clinical, inpatient, community, recoveryfocused) will be considered. Searches will be limited to peer-reviewed journals with grey literature, commentaries, conference abstracts, editorial or opinion pieces excluded.

\section{Eligibility criteria}

To be included in this review, studies must:

1. Involve adult participants ( $\geq 18$ years).
2. Involve participants diagnosed with any mental illness or mental health condition (eg, depression, schizophrenia).

3. Involve any form of goal planning.

4. Report on the impact of goal planning on health outcomes.

5. Report on the experiences of health practitioners and/ or service users involved in goal planning processes.

All studies meeting the above criteria and published in the English language will be considered for inclusion, regardless of study design.

Studies will be excluded if they focus on carer experiences rather than the service user or practitioner or if they include a mixed population (eg, report on a range of disabilities) where data specific to those experiencing mental illness cannot be extracted.

\section{Screening}

Database search results will be downloaded and saved into EndNote where duplicates will be removed, and titles screened for relevance by one member of the research team. Remaining articles will be shared with another member of the research team using the Covidence systematic review software program. Abstracts will be independently screened regarding eligibility for study inclusion by two members of the research team. Articles that are deemed irrelevant by both reviewers will be excluded, those with a discrepancy will be included in the full-text search. Full texts will be obtained for all remaining articles and screened independently by two members of the research team to determine eligibility for study inclusion. Reasons for exclusion will be recorded and any disagreements will be discussed and resolved through discussion with a third member of the research team.

\section{Data extraction}

The following information from each eligible article will be extracted and recorded in a tabular format: author, publication year, country, study design, aims, study setting, sample characteristics (eg, diagnosis, age, gender), goal planning method, healthcare practitioner population(s), 
description of intervention(s), goal planning outcome measure(s), service user results and practitioner results.

\section{Quality assessment}

As this systematic review aims to include all studies relevant to the research questions, it is anticipated that included literature will employ a broad range of study designs (eg, qualitative, quantitative and mixed method studies). The inclusion of a range of studies can provide a deeper understanding of the impact of contextual factors and allow for analysis of the diversity of effect across a range of populations, interventions and settings. ${ }^{31}$ However, appraising the methodological quality of studies with diverse designs remains challenging. ${ }^{32}$ As such, it is only after the completion of data extraction that identification of the most appropriate quality assessment tool(s) will be possible. The Mixed Methods Appraisal Tool (MMAT) may be used if the resulting literature includes diverse study designs, allowing for the use of one tool to critically appraise quantitative descriptive studies, randomised controlled trials, non-randomised studies, qualitative studies and mixed methods studies. ${ }^{31}$ Alternatively, if the included studies are largely of a quantitative or qualitative design, or include study designs not assessed by the MMAT, appropriate tools will be chosen to accurately assess these study designs (eg, Critical Appraisal Skills Programme tools).

\section{Data synthesis}

Initially, data will be extracted and presented in a tabular format for descriptive synthesis. As it is likely that studies identified for this review will include diverse methodologies, it is anticipated that a mixed methods synthesis will be required. ${ }^{33}$ Mixed methods synthesis allows for a holistic understanding of the topic by integrating findings from multiple sources of data and study types. ${ }^{34}$ As this review aims to explore several research questions, a parallel-results convergent design for synthesising the data is likely to be the most relevant. ${ }^{31}$ This synthesis design allows qualitative and quantitative evidence to be analysed separately and then brought together at the discussion and recommendation stage. ${ }^{31}$ Quantitative and qualitative data synthesis methods will be informed by the type of studies meeting the eligibility inclusion criteria. The research team will discuss which methods best suit the included studies and which answer the research questions prior to data synthesis, and it is anticipated that a range of methodologies may be needed. The literature review and synthesis will be completed in 2021.

\section{Patient and public involvement}

This systematic review was developed and coauthored by a research team including health service researchers, health practitioners and a lived experience researcher, thereby integrating a range of expertise and perspectives.

\section{Ethics and dissemination}

Ethics approval is not required for this study as only published studies with non-identifiable data will be used.
The results of the systematic review will be disseminated through conference presentations and a peer-reviewed manuscript.

\section{CONCLUSION}

Although goal planning is used extensively within mental health service delivery, there is limited understanding of the types of goals being set, experiences of service users and practitioners, and the effectiveness of goal planning as a mental health intervention. This systematic review will explore, present and analyse the evidence relating to the use of goal planning in mental health service delivery, thereby providing guidance on how goal planning should be used in mental health service delivery and highlighting gaps in the current literature.

\section{Twitter Victoria Stewart @v_stewart001 and Sarira El-Den @SariraEI_Den}

Acknowledgements We acknowledge the support of Ms Michelle DuBroy (academic librarian, Griffith University) for assistance with developing the search strategy for this systematic review.

Contributors All authors have contributed to the manuscript. The protocol was developed by VS, SSMM and AJW with HR reviewing the research questions, search strategy and terminology. VS wrote the first draft of the manuscript. SSMM, AJW, HR, SE-D and RN contributed to writing and reviewing the manuscript. All authors have reviewed and approved the final version of the manuscript and will be involved in conducting the systematic review.

Funding The authors have not declared a specific grant for this research from any funding agency in the public, commercial or not-for-profit sectors.

Competing interests None declared.

Patient and public involvement Patients and/or the public were involved in the design, or conduct, or reporting, or dissemination plans of this research. Refer to the Methods section for further details.

Patient consent for publication Not required.

Provenance and peer review Not commissioned; externally peer reviewed.

Open access This is an open access article distributed in accordance with the Creative Commons Attribution Non Commercial (CC BY-NC 4.0) license, which permits others to distribute, remix, adapt, build upon this work non-commercially, and license their derivative works on different terms, provided the original work is properly cited, appropriate credit is given, any changes made indicated, and the use is non-commercial. See: http://creativecommons.org/licenses/by-nc/4.0/.

\section{ORCID iDs}

Victoria Stewart http://orcid.org/0000-0003-2892-2288

Sara Sinclair McMillan http://orcid.org/0000-0003-3427-4467

Helena Roennfeldt http://orcid.org/0000-0002-4569-1226

Sarira El-Den http://orcid.org/0000-0001-7500-3351

Ricki Ng http://orcid.org/0000-0002-1334-6487

Amanda J Wheeler http://orcid.org/0000-0001-9755-674X

\section{REFERENCES}

1 Locke EA. Toward a theory of task motivation and incentives. Organ Behav Hum Perform 1968;3:157-89.

2 Cairns AJ, Kavanagh DJ, Dark F, et al. Goal setting improves retention in youth mental health: a cross-sectional analysis. Child Adolesc Psychiatry Ment Health 2019;13:31-8.

3 Levack WMM, Weatherall M, Hay-Smith EJC, et al. Goal setting and strategies to enhance goal pursuit for adults with acquired disability participating in rehabilitation. Cochrane Database Syst Rev 2015;2015:CD009727.

4 Scobbie L, Dixon D, Wyke S. Goal setting and action planning in the rehabilitation setting: development of a theoretically informed practice framework. Clin Rehabil 2011;25:468-82. 
5 Bandura A, Freeman W, Lightsey R. Self-Efficacy: the exercise of control. Springer, 1999.

6 Schwarzer R. Self-Efficacy: thought control of action. Washington: Hemisphere Pub. Corp, 1992.

7 Locke EA, Latham GP. Building a practically useful theory of goal setting and task motivation. A 35-year odyssey. Am Psychol 2002:57:705-17.

8 Siegert RJ, Levack WM. Rehabilitation goal setting: theory. practice and evidence: CRC press 2015

9 Holliday RC, Ballinger C, Playford ED. Goal setting in neurological rehabilitation: patients' perspectives. Disabil Rehabil 2007;29:389-94.

10 Bovend'Eerdt TJH, Botell RE, Wade DT. Writing smart rehabilitation goals and achieving goal attainment scaling: a practical guide. Clin Rehabil 2009;23:352-61.

11 McGuire AB, Lysaker PH, Wasmuth S. Altered Self-Experience and goal setting in severe mental illness. Am J Psychiatr Rehabil 2015;18:333-62.

12 Slade M, Amering M, Farkas M, et al. Uses and abuses of recovery: implementing recovery-oriented practices in mental health systems. World Psychiatry 2014;13:12-20.

13 Lakeman R. Mental health recovery competencies for mental health workers: a Delphi study. J Ment Health 2010;19:62-74.

14 Anthony WA. Recovery from mental illness: the guiding vision of the mental health service system in the 1990s. Psychosocial Rehabilitation Journal 1993;16:11-23.

15 Andresen R, Oades L, Caputi P. The experience of recovery from schizophrenia: towards an empirically validated stage model. Aust $N$ Z J Psychiatry 2003;37:586-94

16 Biringer E, Davidson L, Sundfør B, et al. Experiences of support in working toward personal recovery goals: a collaborative, qualitative study. BMC Psychiatry 2016;16:426.

17 Jensen SB, Eplov LF, Mueser KT. Participants' lived experience of pursuing personal goals in the Illness Management and Recovery program. International Journal of Social Psychiatry 2020;0:0020764020954471.

18 Levack WMM, Dean SG, Siegert RJ, et al. Purposes and mechanisms of goal planning in rehabilitation: the need for a critical distinction. Disabil Rehabil 2006;28:741-9.

19 Jørgensen K, Rendtorff JD. Patient participation in mental health care - perspectives of healthcare professionals: an integrative review. Scand J Caring Sci 2018;32:490-501.

20 De las Cuevas C, Peñate W. To what extent psychiatric patients feel involved in decision making about their mental health care? relationships with socio-demographic, clinical, and psychological variables. Acta Neuropsychiatr 2014;26:372-81.
21 Lexell EM, Lexell J, Larsson-Lund M. The rehabilitation plan can support clients' active engagement and facilitate the process of change - experiences from people with late effects of polio participating in a rehabilitation programme. Disabil Rehabil 2016;38:329-36.

22 Holliday RC, Cano S, Freeman JA, et al. Should patients participate in clinical decision making? an optimised balance block design controlled study of goal setting in a rehabilitation unit. $J$ Neurol Neurosurg Psychiatry 2007;78:576-80.

23 Dekker J, de Groot V, Ter Steeg AM, et al. Setting meaningful goals in rehabilitation: rationale and practical tool. Clin Rehabil 2020;34:3-12.

24 Hamovitch EK, Choy-Brown M, Stanhope V. Person-Centered care and the therapeutic alliance. Community Ment Health $J$ 2018;54:951-8

25 Franklin $\mathrm{M}$, Lewis S, Willis $\mathrm{K}$, et al. Controlled, constrained, or flexible? how self-management goals are shaped by patient-provider interactions. Qual Health Res 2019;29:557-67.

26 Easter A, Pollock M, Pope LG, et al. Perspectives of treatment providers and clients with serious mental illness regarding effective therapeutic relationships. J Behav Health Serv Res 2016:43:341-53

27 Bee P, Price O, Baker J, et al. Systematic synthesis of barriers and facilitators to service user-led care planning. Br J Psychiatry 2015;207:104-14.

28 Green M, Gordon C. Shared decision making in the treatment of psychosis: should we include whether and when as well as which antipsychotic? Psychiatr Times 2013;30:33.

29 Shamseer L, Moher D, Clarke M, et al. Preferred reporting items for systematic review and meta-analysis protocols (PRISMA-P) 2015: elaboration and explanation. BMJ 2015;349:g7647.

30 Yensen J. PICO search strategies. Online Journal of Nursing Informatics 2013;17:1-5.

31 Hong QN, Gonzalez-Reyes A, Pluye P. Improving the usefulness of a tool for appraising the quality of qualitative, quantitative and mixed methods studies, the mixed methods appraisal tool (MMAT). J Eval Clin Pract 2018;24:459-67.

32 Souto RQ, Khanassov V, Hong QN, et al. Systematic mixed studies reviews: updating results on the reliability and efficiency of the mixed methods appraisal tool. Int J Nurs Stud 2015;52:500-1.

33 Sandelowski M, Voils $\mathrm{Cl}$, Barroso J. Defining and designing mixed research synthesis studies. Res Sch 2006;13:29.

34 Ferguson SL, Kerrigan MR, Hovey KA. Leveraging the opportunities of mixed methods in research synthesis: key decisions in systematic mixed studies review methodology. Res Synth Methods 2020;11:580-93. 